\title{
Ionization of Hydrogen Rydberg Molecules at a Metal Surface
}

\author{
G. R. Lloyd, S. R. Procter, and T. P. Softley \\ Department of Chemistry, University of Oxford, Chemistry Research Laboratory, Mansfield Road, Oxford OX1 3TA, United Kingdom
}

(Received 1 July 2005; published 19 September 2005)

\begin{abstract}
The interaction of a beam of Rydberg molecules with a metal surface is investigated for the first time. Hydrogen molecules in a supersonic expansion are excited to Rydberg states with principal quantum number $n$, in the range 17-22 and are directed at a small angle onto a flat surface of either aluminum or gold. Detection of ions produced when Rydberg electrons tunnel into the metal surface provides information on the interaction between the Rydberg molecules and the surface potential. The experimental results suggest that, when close to the metal surface, the Rydberg molecules undergo a process of surfaceinduced rotational autoionization. It is found that the surface-ionization cross section shows strong resonances as a function of the applied electric field, which are independent of the metal studied.
\end{abstract}

DOI: 10.1103/PhysRevLett.95.133202

The interaction of Rydberg atoms with metallic surfaces and thin metallic films has been the subject of several previous experimental and theoretical studies [1-9]. As the atom approaches the surface the Rydberg electron is subject to fields caused by the presence of image charges in the metal, and thus the physics is closely related to the Stark effect behavior of the atom. It has been shown that the predominant process at sufficiently close distance of approach is the tunneling ionization of the Rydberg electron into the conduction band of the metal. The ability to manipulate the Rydberg electron spatial distribution is an important aspect of these studies. Not only can the mean radius of the Rydberg orbit be adjusted by selection of principal quantum number $\left(\langle r\rangle \approx n^{2} a_{0}\right)$ but the angular distribution of the electron density can also be controlled by selection of specific Stark states in the presence of a field; thus, it is possible to polarize the atom such that the Rydberg electron distribution lies either in front of the ionic core, or trails behind the ionic core, as the atom approaches the surface. Measurements of the field required to pull the resultant ion away from collision with the surface have previously shown that the distance at which ionization occurs is strongly dependent on the principal quantum number, but surprisingly shows little dependence on the angular distribution [10]. The tunneling ionization probability should also be dependent on the long-range surface potential and the degree of surface roughness [2], as well as being sensitive to the presence of adsorbates [4].

The interaction of a Rydberg molecule, such as $\mathrm{H}_{2}$, with a metal surface presents new aspects to the physical problem. First the molecular ion core $\left(\mathrm{H}_{2}{ }^{+}\right.$in this case) possesses vibrational and rotational degrees of freedom and therefore the question arises as to how these might contribute to the interaction with the surface, and the tunneling ionization probability. Second the Rydberg molecule has sufficient energy to undergo dissociation into neutral species $(\mathrm{H}+\mathrm{H})$, and therefore the possibility exists that the collision with the surface could induce predissociation in competition with ionization. There is also a greater chemical interest in using species other than rare gas or alkali
PACS numbers: 34.60.+z, 34.50.Dy

metal atoms for these studies, in that the products of tunneling ionization $\left(\mathrm{H}_{2}{ }^{+}\right)$or dissociation ( $\mathrm{H}$ atoms) could potentially become involved in secondary chemical processes at the surface. More generally, there is a growing interest in the interactions of Rydberg atoms and molecules with other types of surface. On the one hand it has been proposed that Rydberg atoms could be used for nanoscale pattern deposition on semiconductors [11], especially as the spatial distribution of the Rydberg atoms could be controlled using laser fields [11] or inhomogeneous electric field focusing [12].

On the other hand there is substantial evidence for the thermal emission of Rydberg species (atoms, molecules and clusters) from impregnated metal oxide surfaces $[13,14]$, and thus studies such as those initiated in the present work may help us to understand these desorption processes through the applicability of microscopic reversibility.

The experimental setup used in the present work is shown in Fig. 1. A population of $\mathrm{H}_{2}$ Rydberg states is produced in a pulsed, skimmed supersonic expansion using a vacuum ultraviolet (VUV) - ultraviolet double-resonant

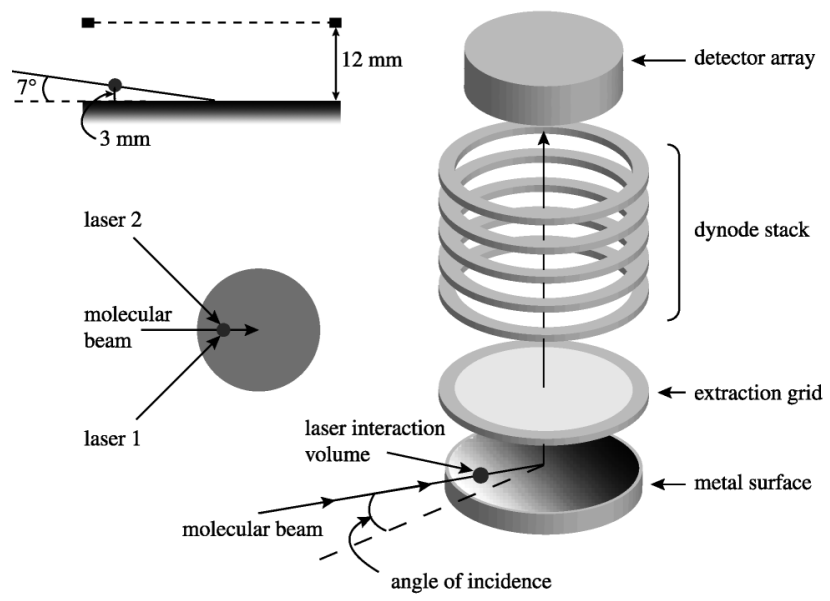

FIG. 1. Experimental setup. 


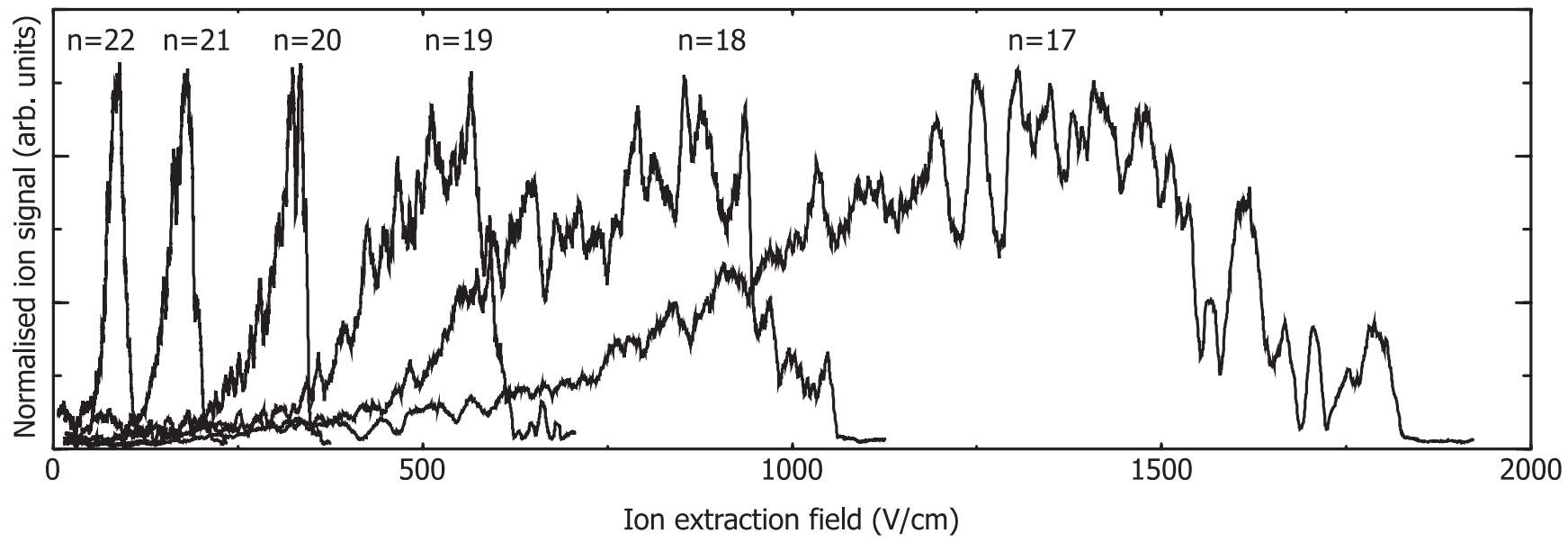

FIG. 2. Surface-ionization signal vs applied extraction field for population of the $n d[2]_{1}$ Rydberg states for a range of values of $n$.

excitation scheme, described in detail elsewhere $[15,16]$. Briefly, VUV radiation $(\lambda=110.81 \mathrm{~nm})$ is obtained by nonresonant frequency tripling of the frequency-doubled output of a Nd:YAG pumped dye laser in a cell containing a $\mathrm{Kr} / \mathrm{Ar}$ mixture (1080/200 mbar). The beam is focused into the vacuum chamber using a lithium fluoride lens $(f=$ $70 \mathrm{~mm})$. The VUV frequency is resonant with the $R(0) B^{1} \Sigma_{u}^{+}\left(v^{\prime}=0\right)-X^{1} \Sigma_{g}^{+}\left(v^{\prime \prime}=0\right)$ one-photon transition of para-hydrogen at an excitation energy of $90424.33 \mathrm{~cm}^{-1}$ [17]. Only the $J^{\prime}=1$ rotational level of the $B^{1} \Sigma_{u}^{+}\left(v^{\prime}=0\right)$ intermediate state is populated. The UV beam, which is tunable from $291-296 \mathrm{~nm}$, is provided by the frequency-doubled output of a second dye laser pumped by the same Nd:YAG. The UV frequency is chosen to be resonant with the one-photon transitions from the intermediate state to the Rydberg series converging on the ionic thresholds associated with the $v^{+}=0$, $N^{+}=0$, and 2 levels of the $X^{2} \Sigma_{g}^{+}$ground ionic state $\left(v^{+}\right.$ and $N^{+}$are, respectively, the vibrational and rotational angular momentum quantum numbers of the ionic levels). The $v^{+}=0, N^{+}=0$ threshold lies at an energy $124417.5(24) \mathrm{cm}^{-1}$ above the ground state [18]. Since the intermediate has ungerade electronic symmetry the UV excitation step can access only the even- $l$ Rydberg series, predominantly $n s$ and $n d$, with total angular momentum $J=0,1$, or 2 . The work detailed here focuses mainly on the $J=1 n d$ Rydberg series converging to the $N^{+}=2$ ionic threshold energy, labeled $n d[2]_{1}$. The polarizations of the two lasers are mutually perpendicular, and are both perpendicular to the field direction, resulting in excitation of levels with $M_{J}=0$ or \pm 2 .

The Rydberg-surface collision experiments are performed in a high vacuum sample chamber with a base pressure of $\sim 8 \times 10^{-8} \mathrm{mbar}$, rising to $\sim 1 \times 10^{-6} \mathrm{mbar}$ during operation of the pulsed nozzle. The nozzle and the skimmer are arranged such that the resulting beam of $\mathrm{H}_{2}$ molecules is incident upon the horizontally mounted metal surface at an angle of approximately $7^{\circ}$. The intersection between the laser beams and the molecular beam occurs in a region of spatially homogeneous electric field between the metal surface and a mesh grid electrode as shown in Fig. 1. The metal sample is mounted on a precision translation stage allowing vertical control of the surface position. Typically this is chosen so that the Rydberg molecules are excited $3 \mathrm{~mm}$ above the surface such that the surfacemesh separation is $12 \mathrm{~mm}$ (as shown in the inset to Fig. 1). The Rydberg states are excited under nearly field-free conditions, although a small negative potential $(-10 \mathrm{~V})$ is applied to the mesh electrode to separate prompt ions that are formed during laser excitation.

The sample surface was prepared on a three-inch diameter polished $\mathrm{Si}(100)$ wafer by metal vapor deposition forming an aluminum $(99.999 \%)$ layer of $\sim 100 \mathrm{~nm}$ thickness. The resulting surface topography was characterized using an atomic force microscope operated in tapping mode using a silicon probe. The root mean roughness $\left(R_{a}\right)$ of the top surface film was found to be $\sim 2 \mathrm{~nm}$.

To observe $\mathrm{H}_{2}{ }^{+}$ions formed through the Rydberg molecule interaction with the surface, a large variable positive potential is applied to the surface approximately $800 \mathrm{~ns}$ following excitation. For molecules that are ionized close to the surface, the resulting ions will collide with the surface unless the applied potential is sufficiently large to oppose the incident momentum and the image charge attraction of the ion. Above this threshold field, the ions are accelerated through the mesh electrode and are detected by a position sensitive detector comprising a pair of multichannel plates and phosphor screen viewed by either a photomultiplier tube (PMT) or a CCD camera. In addition to surface-induced ionization, the Rydberg molecules may also undergo direct field ionization in the gas phase at higher field strengths. At fields corresponding to the onset of direct ionization, two peaks can be seen in the time-of-flight (TOF) profiles. An early sharp peak is observed $\sim 400 \mathrm{~ns}$ after application of the pulsed field due to ions produced by the direct field ionization process; the arrival time of these ions depends on the pulsed field timing. The second broad signal arises from Rydberg 
molecules ionizing as they reach the surface, demonstrated by the fact that the arrival time is independent of the pulse timing. Thus, the different time-of-flight of the two peaks allows the different ionization mechanisms to be distinguished experimentally. The ions from the two mechanisms also appear at different positions on the detector giving further differentiation of these signals.

Figure 2 shows the variation in the surface-ionization signal as a function of applied ion extraction field for initial population of the $n d[2]_{1}$, Rydberg states with $n=17-22$. For each profile, the decrease of the signal on the high-field side is due to the onset of direct field ionization, which prevents the Rydberg molecules from reaching the surface. It is observed that the low-field onset of the surfaceionization signal occurs at higher fields when states of lower principal quantum number are populated. This reflects the smaller size of the Rydberg electron orbital, and hence the smaller distance from the surface at which tunneling ionization occurs. Simple electrostatic considerations (assuming a free-electron model for the metal and a hydrogenic model for the Rydberg state) allow the calculation in atomic units of the minimum field, $E_{\min }$, required to ensure that an ion escapes the surface and is detected [2]:

$$
E_{\min }=\left(\sqrt{\frac{T_{\perp}}{Z_{i}}}+\frac{1}{2 Z_{i}}\right)^{2},
$$

where the ion is formed at a distance from the surface $Z_{i}$, and has a kinetic energy perpendicular to the surface $T_{\perp}$. Through the link between ionization distance and applied field given in Eq. (1), the profiles in Fig. 2 can be considered to show how the tunneling ionization probability varies with distance from the surface, after taking into account the spread of incident kinetic energies. An interesting feature of the data is that the onset of the surface signal occurs at much lower fields than would be predicted by Eq. (1) assuming that surface ionization occurs at around $Z_{i}=4.5 n^{2} a_{0}$ as found previously for xenon atoms [2] and using the principal quantum numbers assigned to the initially excited $N^{+}=2$ levels. In fact, onset fields are more characteristic of the behavior expected of energetically nearby $N^{+}=0$ Rydberg states of higher principal quantum number converging to the lowest-lying rotational threshold. This suggests that the overall ionization process involves a complete transfer of energy from the $\mathrm{H}_{2}^{+}$ioncore rotation to the Rydberg electron. This "surfaceinduced autoionization" is closely related to the fieldinduced autoionization observed previously for ionization of these same states in the gas phase $[15,16]$. This interpretation is supported by Fig. 3, in which the ionization profiles have been converted so that the horizontal scale is in units of $Z_{i} / n^{2}$ with $Z_{i}$ determined from Eq. (1). If the tunneling ionization distance were to scale as $n^{2}$ (which is to be expected from the scaling of the Rydberg orbital radius) then the data for all principal quantum numbers should be represented by a single curve. It is clear from Fig. 3(a) that this is not the case. However, as shown in

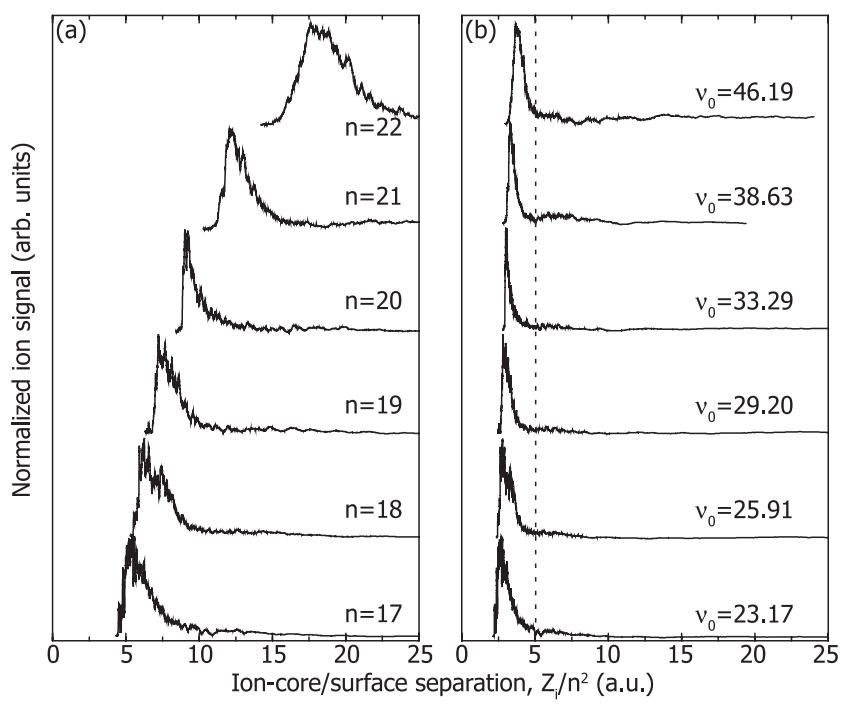

FIG. 3. (a) Surface-ionization profiles for $n=17-22$ Rydberg states at the aluminum surface plotted as a function of $Z_{i} / n^{2}$ using Eq. (1) assuming $T_{\perp}=1.5849 \times 10^{-22}$ J. (b) Same data as in (a), except that $\nu_{0}$ [Eq. (2)] is used in calculating the horizontal axis rather than $n$.

Fig. 3(b) it is found that the ionization distance does scale with $\nu_{0}^{2}$, where $\nu_{0}$ is an effective principal quantum number calculated with respect to the $N^{+}=0$ threshold using the equation

$$
E=I\left(N^{+}=0\right)-\frac{\Re}{\nu_{0}^{2}} .
$$

Figure 3(b) also shows that ionization occurs over a distance range of $Z_{i} \sim 3-5 \nu_{0}^{2} a_{0}$ from the surface which is in good agreement with the range found in previous experiments by Nordlander and co-workers for Xe atoms [2].

Perhaps an even more interesting and unexpected feature of the data shown in Figs. 2-4 is that the profiles are not a smoothly changing function of applied field, but instead show strong resonance behavior that was not apparent in previous measurements on atomic systems [2]. The experimental reproducibility of the resonances is illustrated by the results of two separate measurements illustrated in Fig. 4(a). No changes were observed in this effect under variation of field step size, laser intensity, or the position at which the beam strikes the surface. At the present time the origin of this structure, and whether it is a specific feature of the molecular nature of the Rydberg core, remains to be firmly established. Figure 4(b) shows a comparison of the surface-ionization profiles for the $N^{+}=2, n=20$ state recorded for both an aluminum and an evaporated gold surface $\left(99.999 \%\right.$ purity, $R_{a}=1 \mathrm{~nm}$ ). The two profiles are very similar and share many of the same features; thus the resonant behavior appears to be independent of the type of metal, although there may be subtle differences that are not distinguished within the signal-to-noise ratio.

A plausible explanation for the observed structure would be that the fields at which surface ionization is enhanced 


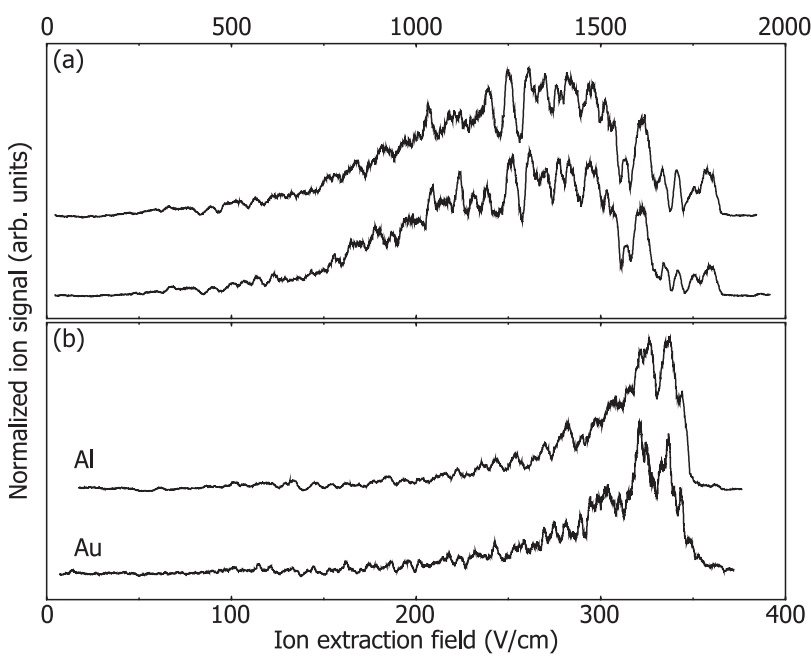

FIG. 4. (a) Reproducibility of the surface-ionization signal vs extraction field for population of the $17 d[2]_{1}$ state in two separate field scans. (b) Comparison of surface-ionization profiles for the $20 d[2]_{1}$ state using evaporated gold and evaporated aluminum surfaces.

correspond to avoided crossings in the Stark map between the initially populated $n d[2]_{1}$ level and the nearby $N^{+}=0$ Stark manifolds [for the $17 d[2]_{1}$ level these would be the $n=21$ and 22 manifolds; see Fig. 8 of Ref. [16] ]. At these fields the degree of $N^{+}=0$ character in the Rydberg molecule wave function could be enhanced leading to a greater probability of surface-induced autoionization.

At the present time there are no theoretical predictions or expectations for this new case of a Rydberg molecule interacting with a surface. In principle, the problem can be approached by adaptation of the complex scaling method described by Nordlander and co-workers in Refs. [5,6,19]. The basis set would need to be extended to include the rotational wave function of the $\mathrm{H}_{2}^{+}$core and the couplings between the rotational channels could be derived from quantum defects as we described in Ref. [16]. It should be noted that the extension of basis set multiplies the required number of functions by an order of magnitude. In addition, a full treatment would also require inclusion of predissociation [which could also be tackled using a complex scaling approach [20]], and also determination of the evolution of the Rydberg wave function as it passes a multitude of avoided crossings in the Stark map as the field is ramped up prior to surface interaction. It is hoped that the present work will stimulate detailed theoretical investigations of this type.

In summary, these first studies of the interaction of Rydberg molecules with a metal surface show clearly that the presence of rotational degrees of freedom does have a significant effect on the tunneling ionization process. Thus there is a specific molecular effect to be observed. Future work will aim to investigate whether other molecular effects can occur, such as surface-induced pre- dissociation of the Rydberg molecules, and whether the branching ratio for such processes can be controlled by choice of field and spectroscopic selection. In the longer term the interaction of Rydberg molecules with surfaces could offer new opportunities for studying reactive processes. For example, interaction of a Rydberg molecule with an adsorbate could proceed via the transfer of the Rydberg electronic energy into the breaking of a chemical bond, followed by secondary reaction with the incident molecule. The possibility to control not only the electronic energy, but also the spatial divergence and the velocity of impact of the Rydberg beams [16] would also be a valuable feature of such experiments.

The authors are grateful to Dr. R. M. J. Jacobs for his advice and assistance in the preparation and characterization of the sample surfaces, to Dr. Y. Yamakita for his work on the design of the vacuum chamber, and to the EPSRC for financial support.

[1] S. A. Deutscher, X. Yang, and B. Joachim, Phys. Rev. A 55, 466 (1997).

[2] S. B. Hill, C. B. Haich, Z. Zhou, P. Nordlander, and F. B. Dunning, Phys. Rev. Lett. 85, 5444 (2000).

[3] C.A. Kocher and C. R. Taylor, Phys. Lett. A 124, 68 (1987).

[4] G.E. McCown, C.R. Taylor, and C.A. Kocher, Phys. Rev. A 38, 3918 (1988).

[5] P. Nordlander and J.C. Tully, Surf. Sci. 211-212, 207 (1989).

[6] P. Nordlander, Phys. Rev. B 53, 4125 (1996).

[7] C. Fabre, M. Gross, J.M. Raimond, and S. Haroche, J. Phys. B 16, L671 (1983).

[8] K. Ganesan and K. T. Taylor, J. Phys. B 29, 1293 (1996).

[9] U. Thumm, P. Kurpick, and U. Wille, Phys. Rev. B 61, 3067 (2000).

[10] F. B. Dunning, H. R. Dunham, C. Oubre, and P. Nordlander, Nucl. Instrum. Methods Phys. Res., Sect. B 203, 69 (2003).

[11] N. A. Nguyen, B. K. Dey, M. Shapiro, and P. Brumer, J. Phys. Chem. A 108, 7878 (2004).

[12] O. Kritsun and H. Metcalf, Bull. Am. Phys. Soc. 47, 109 (2002).

[13] L. Holmlid, J. Phys. Chem. A 102, 10636 (1998).

[14] J. Wang and L. Holmlid, Chem. Phys. 261, 481 (2000).

[15] S. R. Procter, Y. Yamakita, F. Merkt, and T. P. Softley, Chem. Phys. Lett. 374, 667 (2003).

[16] Y. Yamakita, S. R. Procter, A. L. Goodgame, T. P. Softley, and F. Merkt, J. Chem. Phys. 121, 1419 (2004).

[17] A. A. Radzig and B. M. Smirnov, Reference Data on Atoms, Molecules, and Ions, Springer Ser. Chem. Phys. Vol. 31 (Springer, New York, 1985).

[18] J. M. Gilligan and E.E. Eyler, Phys. Rev. A 46, 3676 (1992).

[19] J. Braun and P. Nordlander, Surf. Sci. 448, L193 (2000).

[20] I. D. Petsalakis, G. Theodorakopoulos, and R. J. Buenker, J. Chem. Phys. 119, 2004 (2003). 